\title{
Decoding Synchronized Oscillations within the Brain: Phase-Delayed Inhibition Provides a Robust Mechanism for Creating a Sharp Synchrony Filter
}

\author{
Mainak Patel ${ }^{* 1}$ and Badal Joshi ${ }^{\dagger 2}$ \\ ${ }^{1}$ Mainak Patel, Mathematics Department, Duke University, Box 90320, Durham, NC \\ 27708-0320, Phone: 919.660.2800, Fax: 919.660.2821 \\ ${ }^{2}$ Badal Joshi, School of Mathematics, University of Minnesota, 127 Vincent Hall, 206 \\ Church St. SE, Minneapolis, MN 55455
}

\begin{abstract}
The widespread presence of synchronized neuronal oscillations within the brain suggests that a mechanism must exist that is capable of decoding such activity. Two realistic designs for such a decoder include: 1) a read-out neuron with a high spike threshold, or 2) a phase-delayed inhibition network motif. Despite requiring a more elaborate network architecture, phase-delayed inhibition has been observed in multiple systems, suggesting that it may provide inherent advantages over simply imposing a high spike threshold. In this work, we use a computational and mathematical approach to investigate the efficacy of the phase-delayed inhibition motif in detecting synchronized oscillations. We show that phase-delayed inhibition is capable of creating a synchrony detector with sharp synchrony filtering properties that depend critically on the time course of inputs. Additionally, we show that phase-delayed inhibition creates a synchrony filter that is far more robust than that created by a high spike threshold. Keywords: phase-delayed inhibition, feedforward inhibition, time-delayed inhibition, synchrony filter, neuronal networks, neuronal network motifs, mathe-
\end{abstract}

*mainak@math.duke.edu

†joshi@umn.edu matical neuroscience, synchronized oscillations, network structure and function

\section{Introduction}

Within the brain, synchronized oscillations are ubiquitous, as evident from local field potential (LFP), electroencephalogram (EEG), and other recordings obtained from diverse species throughout the animal kingdom. In fact, networks of neurons can be found within the brains of all manner of organisms, from insects and fish to birds and mammals, which exhibit synchronized and periodic spiking within physiologically meaningful dynamical regimes (e.g., see Eckhorn, 1994; Friedrich et al., 2004; Gray, 1994; Laurent and Davidowitz, 1994; Marthy and Fetz, 1992; Sridharan et al., 2011). In some instances, such as thalamocortical oscillations during sleep (Steriade, 2006), neuronal oscillations are coordinated on a global scale and may not play a direct role in information processing. In many instances, however, synchronized oscillations are localized and modulated by external stimuli. The overwhelming presence of stimulusdependent, periodic, coherent activity within a vast array of brain areas implies that synchronized oscillations may be a strategy commonly employed by neuronal networks to encode and relay information. 
In order for the brain to use synchronized oscillations as a coding tool, a neural mechanism must exist that is capable of decoding the activity of a population of cells representing information by firing in a coherent and periodic fashion. There are two simple, biologically plausible mechanisms for creating such a decoder: 1) a read-out neuron with a high spike threshold (relative to the strength of individual inputs), and 2) a read-out neuron that receives phase-delayed inhibition.

A decoder with a high threshold detects synchronized oscillations by virtue of the fact that a large proportion of its inputs (the encoders) must fire in unison in order for the decoder to cross spike threshold, and hence this decoder will be active during an oscillation cycle only if this condition is satisfied (figure 1A). An example of a high threshold synchrony decoder can be found in the auditory system; each octopus cell within the ventral cochlear nucleus receive convergent input from at least 60 auditory nerve fibers. Octopus cells have low input resistance, short membrane time constants, and a relatively high spike threshold. These cells therefore have brief temporal integration windows $(\sim 1 \mathrm{~ms})$ and act as coincidence detectors of their input - an octopus cell will fire only if a significant fraction of its presynaptic auditory nerve fibers spike synchronously (see Oertel et al., 2000 for a review).

Phase-delayed inhibition operates in a different manner. The neural architecture underlying phasedelayed inhibition consists of a group of neurons (the encoders) that provide excitation to a read-out neuron (the decoder), but en-route to the read-out cell the axons of the encoders send collaterals to a group of inhibitory interneurons, which in turn provide potent inhibitory input to the read-out cell (figure 1B). As a consequence of synaptic transmission delay (or via some other delay-generating mechanism), each excitatory input to the decoder is followed, with a characteristic temporal lag, by an inhibitory input. The intuition behind phase-delayed inhibition is clear; if the encoders spike haphazardly, then the inhibitory interneurons follow accordingly, and the read-out neuron remains covered in a perpetual blanket of inhibition, unable to respond to the encoders. If, on the other hand, the encoders fire synchronously,
A)

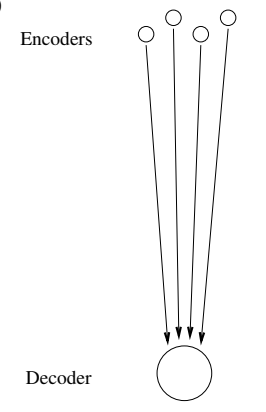

B)

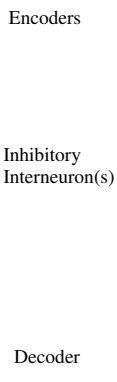

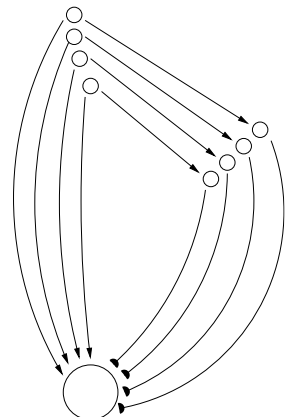

Figure 1: Schematic of network architecture needed to implement synchrony decoding with a high spike threshold versus phase-delayed inhibition. A) High spike threshold decoder. A set of excitatory encoder neurons innervate a decoder neuron with a high spiking threshold relative to the strength of encoder inputs. B) Phase-delayed inhibition decoder. A set of excitatory encoder neurons innervate a set of inhibitory interneurons as well as sending convergent input to a decoder neuron. The decoder neuron also receives input from the inhibitory interneurons. Each excitatory encoder spike is followed, with a slight delay, by an inhibitory interneuron spike.

then the read-out neuron will receive aggregate excitation followed by pooled inhibition, allowing the read-out cell to respond to the encoders within the window of excitation. This process can be repeated within each cycle of oscillation, enabling the decoder neuron to behave as a synchrony filter - the readout cell fires if the encoders spike synchronously but remains quiescent if their spiking is discordant.

Within the brain, anatomical and physiological studies have led to the proposal of phase-delayed inhibition as a means to decode synchronous spiking within a neuronal population in multiple systems (see Real Neural Systems section of Discussion), and several existing models have examined the physiological significance of phase-delayed inhibition (see Other Modeling Work section of Discussion). However, the precise computations performed by the phase-delayed inhibition architecture have yet to be rigorously investigated. In this work, we seek to fill this void. We 
begin by deconstructing the idea into its most mathematically simplified form, and we then verify the results of our simplified scheme by simulating excitatory and inhibitory inputs to an integrate-and-fire model neuron as well as deriving analytic results on neuronal dynamics. We conclude that, within the proper dynamical regime, phase-delayed inhibition can impose a sharp synchrony threshold on the inputs to a read-out cell, and hence can serve as a powerful device to allow a decoder to discard inputs falling below this threshold level of synchrony. Furthermore, we show that phase-delayed inhibition allows for the creation of a synchrony detector that is more robust to noise than a synchrony detector created using a high spike threshold (without inhibition).

\section{Results}

\subsection{Model of Encoder and Interneu- ron Spikes}

In accordance with the diagram presented in figure $1 \mathrm{~B}$, we constructed a network consisting of $n$ encoder neurons, $n$ inhibitory interneurons, and a single read-out neuron (i.e., decoder neuron). In all investigations, the number of inhibitory interneurons was kept equal to the number of encoders, with the number of each given by $n$ (this common number of encoders and interneurons $n$ was varied in some investigations). In order to formulate the problem of phasedelayed inhibition in a mathematically tractable and intuitively transparent manner, we described the activity of the encoders and inhibitory interneurons using step functions distributed over a $T$ ms period. A spike of the $k^{t h}$ encoder cell occurring at time $\tau$ was described by a step function taking the value 0 until time $\tau$, the value $\frac{\alpha}{n}$ from time $\tau$ to time $\tau+c$, and the value 0 from time $\tau+c$ onwards. If the $k^{\text {th }}$ encoder fired at time $\tau$, then a spike was elicited from the $k^{\text {th }}$ inhibitory neuron at time $\tau+d$, which was described by a step function taking the value 0 until time $\tau+d$, the value $\frac{-\beta}{n}$ from time $\tau+d$ to time $\tau+d+h$, and the value 0 from time $\tau+d+h$ onwards. The total amplitude of excitation, in the case that all encoders fired in unison, was therefore given by $\alpha$ (independent of $n$ ). If all inhibitory neurons fired concordantly, the net magnitude of inhibition was given by $\beta$, a parameter which was varied in our simulations. We performed simulations with different values of the synaptic delay $d$ and found similar results for all reasonable values; to simplify subsequent analysis, we fixed the delay at the value $d=3 \mathrm{~ms}$. In agreement with the approximate time course of fast excitatory synapses within the brain, the length of excitation was fixed at $c=3 \mathrm{~ms}$ (Kleppe and Robinson, 1999; Loring and Zigmond, 1988; Thany, 2010; Titz and Keller, 1997; Zhou and Hablitz, 1998). The inhibitory time course $h$ was varied during our investigations (figure 2). The net input to the read-out neuron (i.e., decoder neuron) as a function of time was given by the sum of the $2 n$ step functions describing the activity of the $n$ encoders and $n$ inhibitory neurons.

To test the efficacy of phase-delayed inhibition in decoding coherent oscillations, we set an oscillation frequency $\omega \mathrm{Hz}$ and simulated varying levels of synchrony among the input elements. If $\omega \mathrm{Hz}$ corresponded to a period of $T \mathrm{~ms}$, then each encoder cell was designated to spike exactly once during each $T$ ms period. A synchrony level of 0 (complete decoherence) was simulated by evenly distributing the phases of the $n$ encoder spikes throughout each $T$ ms period, while a synchrony level of 1 (perfect synchrony) was simulated by precisely aligning the spike times of each encoder within every $T$ ms period. Intermediate values of synchrony ranging from 0 to 1 were simulated by evenly distributing encoder spike times throughout progressively smaller time windows within each $T$ ms period (with window size shrinking linearly as a function of synchrony from $T$ to $0 \mathrm{~ms}$ ). The distribution of spike phases throughout a given time window was uniform and deterministic (i.e., not random).

\subsection{Simplified Decoder Model}

Our simplified decoder model was constructed by imposing a numerical threshold on the sum of the $2 n$ step functions describing the activity of the $n$ encoders and $n$ inhibitory neurons. Within our simplified decoder model, we fixed the strength of excitation at $\alpha=1$ and we designated the read-out 


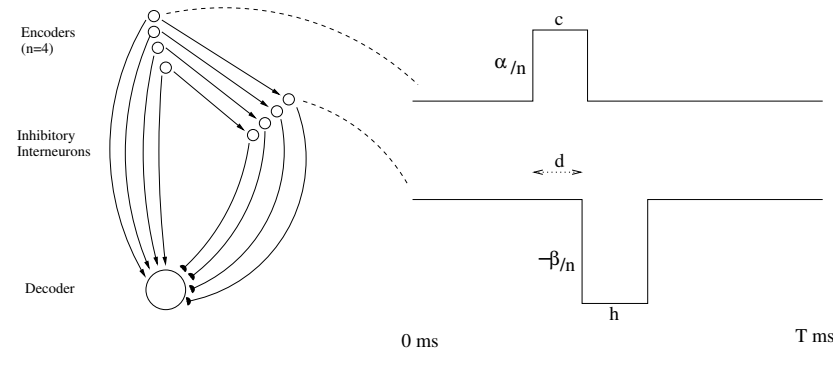

Figure 2: Description of encoder and interneuron spikes as step functions. Within each $T$ ms oscillation period, each of the $n$ encoders was designated to have spiked once, with spike times distributed throughout the $T$ ms period. If an encoder spiked at time $\tau$, then the spike was described by a step function taking the value $\frac{\alpha}{n}$ from time $\tau$ to time $\tau+c$ and the value 0 elsewhere. The corresponding inhibitory interneuron was designated to have spiked at time $\tau+d$, with the spike described by a step function taking the value $\frac{-\beta}{n}$ from time $\tau+d$ to time $\tau+d+h$ and the value 0 elsewhere. We fixed $d=3$, while $\beta$ and $h$ were varied during our simulations. For our simplified decoder model, we fixed $\alpha=1$ and summed the $2 n$ step functions describing the activity of the $n$ encoders and $n$ interneurons; we fixed a numerical threshold $\Theta=0.05$, and the activity of the decoder was measured as the amount of time the step function sum spent above the threshold $\Theta$.

cell (i.e., decoder neuron) as being active (i.e., firing action potentials) when the net input exceeded a particular threshold value $\Theta$. Since the amplitude of each encoder input was normalized (and given by $\frac{\alpha}{n}$, with $\alpha=1$ ), the threshold satisfied $0 \leq \Theta \leq 1$, with the value of $\Theta$ denoting the fraction of encoder cells that were required to be synchronously active in order to induce spiking in the read-out neuron. The activity of the decoder neuron was measured as the total amount of time spent above the threshold $\Theta$.

A high value for $\Theta$ would clearly require a large proportion of encoders to fire concurrently in order to push the read-out cell above threshold, regardless of the presence of inhibition. Thus, phase-delayed inhibition is likely to be most useful as a synchrony filter for a relatively low threshold. For an oscillation frequency of $\omega=20 \mathrm{~Hz}$, a threshold of $\Theta<0.35$ was required to ensure that in the absence of inhibition, asynchronous encoder activity was capable of driving the read-out neuron above threshold. For an oscillation frequency of $\omega=50 \mathrm{~Hz}$, the threshold was required to satisfy $\Theta<0.2$, and an oscillation frequency of $\omega=100 \mathrm{~Hz}$ necessitated $\Theta<0.08$. We accordingly fixed the threshold at a value of $\Theta=0.05$.

\subsubsection{Synchrony Filtering}

In figure 3, we set an oscillation frequency of $\omega=50$ $\mathrm{Hz}$ and plot the activity of the read-out neuron (i.e., time spent above threshold) as a function of encoder synchrony $(n=20)$; the amplitude $(\beta)$ and the time course $(h)$ of inhibition vary from panel to panel. When $\beta=0$ (i.e., no inhibition), the read-out cell exhibits high levels of activity for all levels of encoder synchrony. For nonzero values of $\beta$, however, the presence of phase-delayed inhibition creates a sharp synchrony threshold - the read-out neuron remains quiescent until the encoders reach a particular synchrony level, at which point the activity of the readout cell jumps dramatically. As apparent from figure 3 , the magnitude of inhibition has an effect on the synchrony threshold only over a very small range of $\beta$ values; once $\beta$ exceeds a certain minimal value, the synchrony threshold becomes fixed, and further increases in inhibitory strength are inconsequential. The precise minimum value of $\beta$ after which the synchrony threshold becomes fixed $\left(\beta_{\text {min }}\right)$ depends on other system parameters; however, for all system parameters studied, we found that $\beta_{\text {min }} \leq 8$. The inhibitory time course $(h)$, on the other hand, has a dramatic effect on the synchrony threshold; as $h$ is lengthened, the synchrony threshold systematically shifts towards perfect synchrony. Thus, for a particular set of system parameters, there exists a range of $h$ values between $h_{\min }$ and $h_{\max }$ such that: 1) for $h \leq h_{\min }$, the read-out cell responds to all levels of encoder synchrony, including the maximally asynchronous state where the encoder phases are uniformly distributed over a period; 2) for $h \geq h_{\max }$, the read-out cell is quiet regardless of the level of encoder synchrony; 3 ) by appropriately choosing $h$ within the 
interval $\left(h_{\min }, h_{\max }\right)$, the synchrony threshold of the read-out neuron can be set at any desired value.

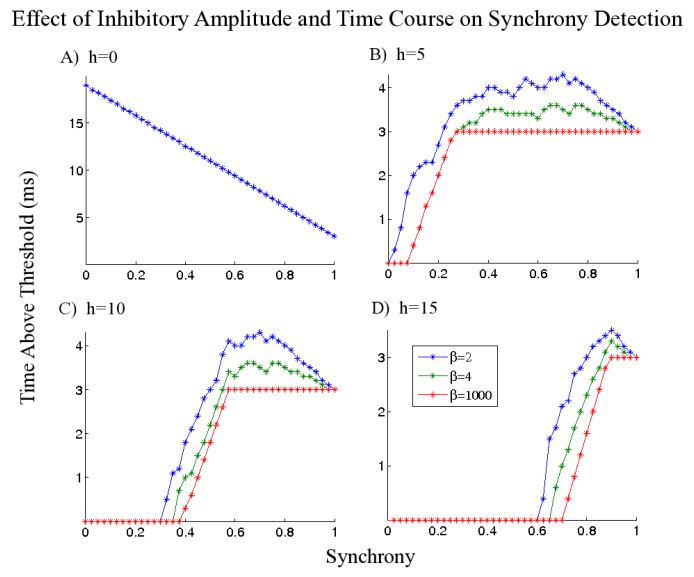

Figure 3: Effect of inhibitory amplitude and time course on synchrony detection using the simplified decoder model. A) Without inhibition, the decoder does not act as a synchrony detector. In $B$ ), $C$ ), and $D)$, the inhibitory time course $h$ is lengthened for different values of the inhibitory amplitude $\beta$; with inhibition, the decoder acts as a sharp synchrony filter, responding only to inputs exceeding a threshold level of synchrony. Increases in the time course $h$ systematically increase the synchrony threshold, while the amplitude $\beta$ has little effect on synchrony filtering. Encoder number was given by $n=20$, the excitatory ammplitude was fixed at $\alpha=1$, and the oscillation frequency was set at $\omega=50 \mathrm{~Hz}(T=20 \mathrm{~ms})$.

In accordance with this result, we fixed the inhibitory amplitude at a large value $(\beta=1000)$ and plotted the activity of the read-out neuron for oscillation frequencies of $\omega=20 \mathrm{~Hz}$ (figure 4A) and $\omega=100$ $\mathrm{Hz}$ (figure $4 \mathrm{~B}$ ). Figures 3 and 4 show that, regardless of the oscillation frequency, lengthening the inhibitory time scale results in a systematic right-shift of the synchrony threshold. Thus, we conclude that the time scale of inhibition is the primary determinant of the efficacy of phase-delayed inhibition as a synchrony filter, while the inhibitory amplitude has little effect on synchrony filtering.

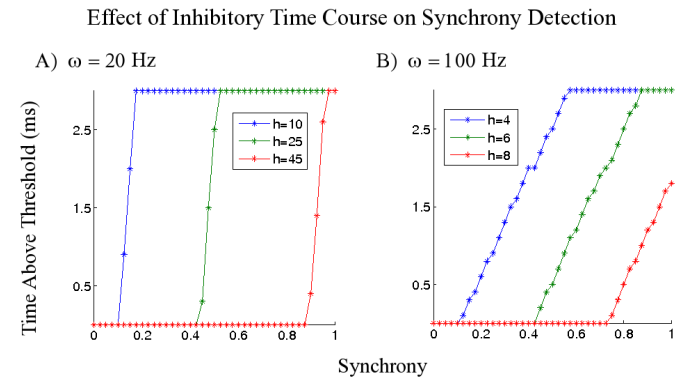

Figure 4: Effect of inhibitory time course on synchrony detection using the simplified decoder model. The inhibitory amplitude $\beta$ is fixed at a large value $(\beta=1000)$, and the response of the decoder is plotted as a function of input synchrony for varying values of the inhibitory time course $h$. For an oscillation frequency of $A) \omega=20 \mathrm{~Hz}(T=50 \mathrm{~ms})$ or $B) \omega=100$ $\mathrm{Hz}(T=10 \mathrm{~ms})$, the decoder responds only to inputs exceeding a threshold level of synchrony, with the value of the synchrony threshold depending on the the time course of inhibition $h$. The encoder number was set at $n=20$ and the excitatory amplitude was fixed at $\alpha=1$.

\subsubsection{Number of Neurons}

In the above simulations, we fixed the number of encoder neurons and number of inhibitory interneurons at $n=20$; however, the question naturally arises as to how many encoders are required in order for phase-delayed inhibition to act as an effective coincidence detector. In order to address this, we fixed the inhibitory amplitude at a large value $(\beta=1000)$ and, for each oscillation frequency, we set a characteristic inhibitory time scale to achieve a synchrony threshold of $\sim 0.5$. In figure 5 , we plot read-out cell activity as a function of synchrony for varying values of $n$ (where $n$ is the number of encoder neurons and inhibitory interneurons), showing that phase-delayed inhibition can create a sharp synchrony threshold using a relatively small number of encoders. In fact, a sharp threshold is observed for neuron numbers as low as $n=5$, with further increases in $n$ exerting little influence on synchrony filtering. This suggests 
that the sum of the $2 n$ step functions (corresponding to the $n$ encoders and $n$ inhibitory neurons) involved in computing read-out cell activity converges rapidly in the large $n$ limit, a limit which we will utilize in subsequent analysis.

\section{Effect of Encoder Number on Synchrony Detection}

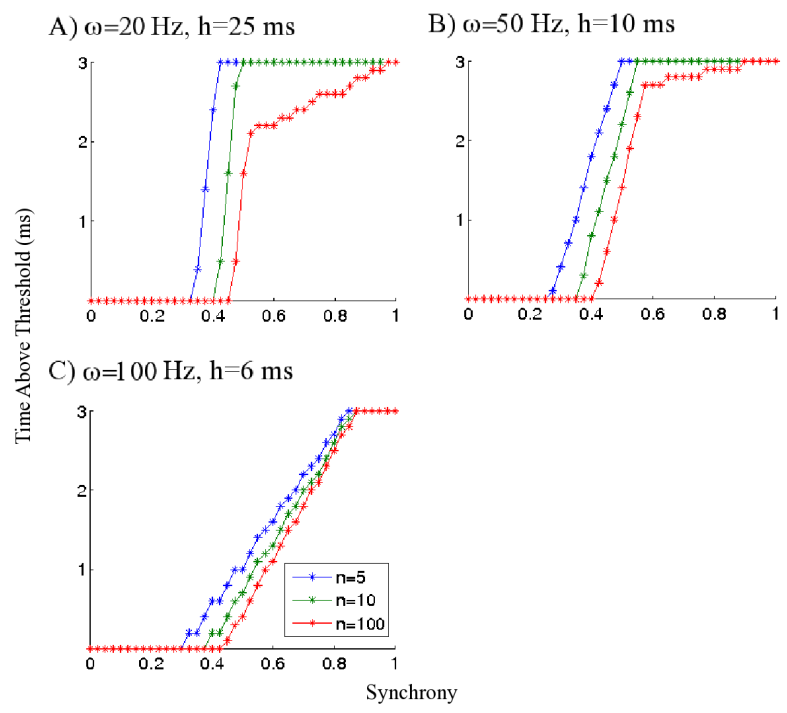

Figure 5: Effect of encoder number on synchrony detection using the simplified decoder model. For oscillation frequencies of $A) \omega=20 \mathrm{~Hz}(T=50 \mathrm{~ms})$, B) $\omega=50 \mathrm{~Hz}(T=20 \mathrm{~ms})$, and $C) \omega=100 \mathrm{~Hz}$ $(T=10 \mathrm{~ms})$, the response of the decoder is plotted as a function of input synchrony for varying encoder numbers $n$. The decoder responds only to inputs exceeding a threshold level of synchrony, and the value of the synchrony threshold rapidly approaches a limiting value for large $n$. The excitatory amplitude was fixed at $\alpha=1$ and the inhibitory amplitude was fixed at $\beta=1000$.

\section{$2.3 \quad$ Integrate-and-Fire Model}

In the above work, we approximate the activity of a read-out neuron by imposing a numerical threshold
$\Theta$ on the sum of the $2 n$ inputs; since the inputs are described by step functions, it is not too surprising that this simplified decoder model would lead to an abruptly rising synchrony-response function for the hypothetical read-out cell. The sum of $2 n$ step functions is a step function, and step functions have flat plateaus and basins rather than sharp peaks and valleys. As synchrony is increased, the input step functions exhibit greater and greater overlap, causing the sum to have higher plateaus and deeper basins. Thus, as soon as synchrony is high enough such that the sum of the step functions rises above $\Theta$, an entire plateau rises above $\Theta$, causing read-out cell activity to jump discontinuously at this synchrony value.

A physiologic read-out neuron, however, exhibits dynamics that are more complex than can be captured by simply imposing a threshold $\Theta$ on the sum of step function inputs. While synaptic inputs to a physiologic read-out neuron are likely to be well approximated by step functions, the membrane potential $V(t)$ of the decoder depends not only on synaptic conductance modulations, but also on the membrane time constant as well as voltage-dependent nonlinearities. Since membrane potential dynamics are not instantaneous, the associated time scale of the response implies that $V(t)$ changes continuously, even when the synaptic inputs are described by discontinuous step functions.

We therefore simulated a physiologic read-out cell using a reduced dimensional integrate-and-fire model. By "reduced dimensional" we mean that the membrane potential $V$ is nondimensional, while time $t$ is in units of milliseconds and $g$ is in units of $\mathrm{ms}^{-1}$. The equation governing membrane potential is given by

$$
\frac{d V}{d t}=-g V+i(t)
$$

where $g$ is the leak conductance, $V_{0}=0$ is the resting potential, and $V_{\text {thresh }}=1$ is the threshold for firing an action potential. In other words, when $V(t)$ approaches $V_{\text {thresh }}$ from below, an action potential is recorded and the membrane potential is reset to $V_{0} . \quad i(t)=\sum_{j=1}^{n} i_{j}(t)$ is the total input current from $n$ excitatory encoder neurons and $n$ inhibitory interneurons. The effective $j$-th input is the sum of 
the excitation from the $j$-th encoder and the inhibition from the corresponding $j$-th interneuron.

$i_{j}(t)= \begin{cases}\alpha / n, & \text { if } t(\bmod T) \in\left(\varphi_{j}, \varphi_{j}+c\right) \\ -\beta / n, & \text { if } t(\bmod T) \in\left(\varphi_{j}+c, \varphi_{j}+c+h\right) \\ 0, & \text { elsewhere }\end{cases}$

where $\alpha, \beta, c, h, T>0, c+h<T$ and $\varphi_{j}$ is the relative phase of the $j$-th neuron with $\varphi_{1}:=0$. For simplicity, we assume the phases to be uniformly distributed over a 'window' of size $w<T$, so that $\varphi_{j}=-(j-1) w / n$. The level of encoder synchrony (ranging from 0 to 1 ) is therefore given by $1-(w / T)$.

Thus, we modeled the synaptic input $i(t)$ as the sum of the $2 n$ step functions representing inputs from the $n$ encoders and $n$ inhibitory neurons, as described in the Model of Encoder and Interneuron Spikes section. Within the simplified decoder model, we simulated a low spike threshold (relative to the net excitatory strength $\alpha=1$ ) by setting $\Theta=0.05$. With the integrate-and-fire decoder, spike threshold is fixed, and hence we set the excitatory strength at a high value $(\alpha=8)$ in order to ensure a spike threshold that is low relative to $\alpha$. Synchrony levels ranging from 0 to 1 were simulated by evenly distributing encoder spike times throughout progressively smaller time windows within each $T \mathrm{~ms}$ period. Using computational simulations, we found that the results obtained above using a numerical threshold $\Theta$ translated relatively unchanged to the case of the integrate-and-fire read-out neuron. The integrate-and-fire decoder behaves as a sharp synchrony filter for relatively small $n$ (as low as $n=5$ ), with further increases in $n$ having little effect on coherence detection (data not shown). Additionally, the time course of inhibition $(h)$ plays a vital role in determining the synchrony filtering properties of the read-out cell, with increases in $h$ yielding systematic right-shifts of the synchrony threshold (figure 6).

However, figure 6 shows that synchrony filtering also appears to be sensitive to the amplitude of inhibition $(\beta)$ - increases in $\beta$ lead to right-shifts of the synchrony threshold, with very large $\beta$ rendering the decoder unable to respond to any inputs. This is different from the results obtained using the simplified model of a numerical threshold $\Theta$, a discrepancy which can be explained by the fact that the time course of the response of an integrate-and-fire neuron directly depends on the amplitude of the input. In other words, given two step function inputs with the same time course but different amplitudes, the membrane potential $V(t)$ takes longer to decay in response to the larger amplitude input than in response to the smaller amplitude input. In terms of $V(t)$, increasing $\beta$ effectively increases $h$ as well. Thus, we conclude that phase-delayed inhibition can serve as a robust mechanism to transform a read-out neuron into a sharp synchrony filter, with the time scale of inhibition (relative to the time scale of excitation) being key in determining the level of synchrony required to induce the decoder to spike.

\subsubsection{Mathematical Analysis}

Above, we presented computational results on the synchrony detection properties of the integrate-andfire decoder for various system parameter values. As shown in figure 6 , we found that the integrate-and-fire decoder behaves as a sharp synchrony filter - below a threshold level of encoder synchrony the decoder does not spike, while above this synchrony threshold the firing rate of the decoder jumps to high values. It follows that the synchrony threshold represents the level of encoder synchrony at which the read-out cell transitions from quiescence to repetitive spiking. In this section, we use this observation to develop analytical results on the synchrony detection properties of the integrate-and-fire decoder.

When the integrate-and-fire decoder is quiescent, an analytical solution to the membrane potential equation can be easily obtained in the case of the limit $n \rightarrow \infty$ (where $n$ is the number of encoders and also the number of interneurons), and this solution is valid as long as the membrane potential of the decoder remains below the spike threshold $\left(V_{\text {thresh }}=1\right)$. Using the analytical solution to the membrane potential equation of the integrate-andfire decoder, we explicitly calculate the synchrony threshold as a function of system parameters, and in particular we examine the relationship between the synchrony threshold of the decoder and the strength of excitation $\alpha$ (with other system parameters fixed). 


\section{Synchrony Detection with an Integrate and Fire Decoder Neuron}

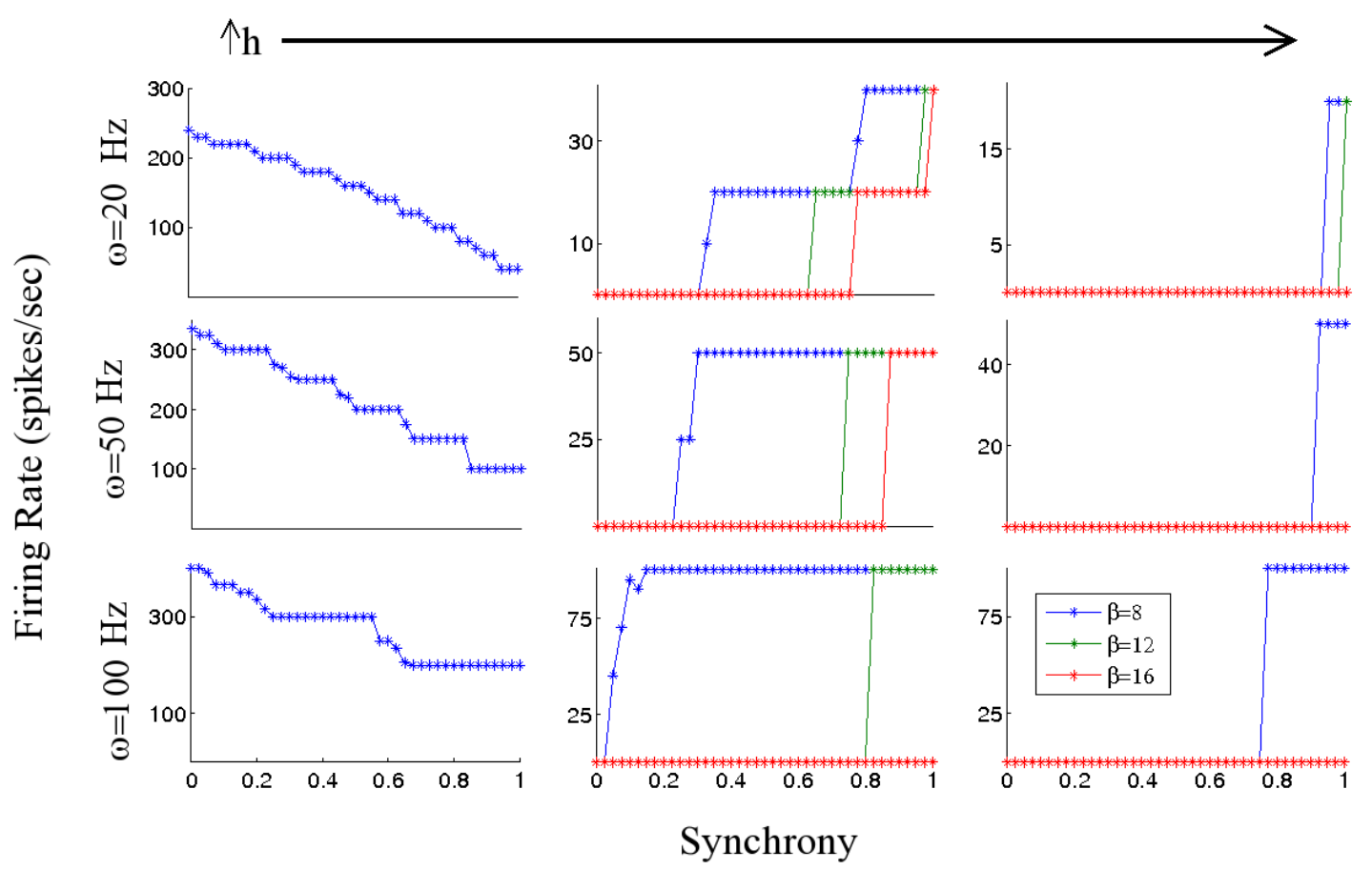

Figure 6: Effect of inhibitory amplitude and time course on synchrony detection using the integrate-and-fire decoder model. Without inhibition (left column), the decoder fires for all levels of input synchrony. In the presence of phase-delayed inhibition, the decoder acts as a sharp synchrony filter, firing only if its inputs exceed a particular threshold level of synchrony. Increases in either the time course $h$ or the amplitude $\beta$ of inhibition yield increases in the value of the synchrony threshold, with very large values of $h$ or $\beta$ rendering the decoder unable to respond for any synchrony level. Encoder number was fixed at $n=20$ and net excitatory amplitude was set to $\alpha=8$. For $\omega=20 \mathrm{~Hz}$, the inhibitory time courses were $h=0,3,15 \mathrm{~ms}$. For $\omega=50 \mathrm{~Hz}$, the inhibitory time courses were $h=0,3,6 \mathrm{~ms}$. For $\omega=100 \mathrm{~Hz}$, inhibitory time courses were $h=0,3,4 \mathrm{~ms}$.

Suppose we fix the system parameters $\beta, c, h, T$, and we set the level of encoder synchrony at a particular value $s$. Since $\alpha$ is the strength of excitation received by the read-out neuron, the read-out neuron will be quiescent for sufficiently low values of $\alpha$, while for sufficiently high values of $\alpha$, the read-out neuron will fire repetitively. For a critical value of $\alpha$ (which we denote by $\alpha_{c}$ ) the readout neuron undergoes a transition from quiescence to repetitive firing mode, as $\alpha$ is increased continuously. We can explicitly calculate $\alpha_{c}$ for different levels of encoder synchrony $s$, and the function $\alpha_{c}(s)$ provides us with insight into the synchrony filtering properties of the system. The details of the calculations are provided in the Supplementary Material.

In order to demonstrate how phase delayed inhibition provides a mechanism for synchrony filtering, we plot in figure $7 \mathrm{~A}$ a graph of the critical value of 
$\alpha\left(\alpha_{c}(s)\right)$ as a function of the level of encoder synchrony $s$ for $\beta=0$ (no inhibition) and $\beta=8$ (with phase delayed inhibition). For $\beta=0, \alpha_{c}(s)$ is almost horizontal; in fact, we find that $\alpha_{c}(0.75)=0.25$ while $\alpha_{c}(1)=0.227$, which implies that $\alpha$ must be finetuned within a narrow band in order for the read-out neuron to act as a synchrony filter. If $\alpha$ is set at a value below this narrow band (i.e., if $\alpha<0.227$ ), then the decoder will be quiescent for all levels of encoder synchrony. If $\alpha$ is set at a value above this narrow band (i.e., if $\alpha>0.25$ ), then the encoder will spike repetitively for all levels of encoder synchrony. For $\alpha$ within this narrow band (i.e., for $0.227 \leq \alpha \leq 0.25$ ), tiny changes in $\alpha$ result in large changes in the synchrony threshold (the level of encoder synchrony at which the transition from no spiking to spiking occurs). Thus, for $\beta=0$, which corresponds to the case of the synchrony detector employing a high spike threshold, the system exhibits a startling lack of robustness - the strength of the excitation $\alpha$ must be set within a very narrow band in order for the readout neuron to have a synchrony threshold, and even within this narrow band, slight fluctuations in $\alpha$ entail radical shifts in the synchrony threshold of the read-out cell. On the other hand, for large, positive $\beta$ (i.e., for a synchrony detector employing phasedelayed inhibition), for instance $\beta=8$, we find that $\alpha_{c}(0.75)=8.58$ and $\alpha_{c}(1)=6.23$; compared to the no inhibition case, there is a larger range of $\alpha$ over which the read-out neuron has a synchrony threshold. For the phase-delayed inhibition case, the system therefore exhibits a greater degree of robustness - for values of $\alpha$ for which a synchrony threshold exists (i.e., for $6.23 \leq \alpha \leq 8.58$ ), small changes in $\alpha$ yield less drastic shifts in the synchrony threshold of the read-out cell.

In the second graph (figure 7B), the strength of inhibition is kept proportional to the strength of the excitation - we plot $\alpha_{c}$ as a function of $s$ for $\beta=\alpha$. When the strength of inhibition $\beta$ is varied with the strength of excitation $\alpha$, we find that synchrony filtering is exceptionally robust. As the nearly vertical portion of the graph in figure 7B suggests, sufficiently high values of $\alpha$ yield a read-out neuron with practically invariant synchrony filtering properties - the synchrony threshold of the read-out cell remains es- sentially untouched even when $\alpha$ is changed considerably.

Thus, synchrony filtering in the no inhibition case $(\beta=0)$ exhibits a delicate sensitivity to $\alpha$, while for constant (nonzero) $\beta$ the synchrony threshold is more robust to fluctuations in the strength of excitation $\alpha$. On the other hand, for $\alpha=\beta$ the synchrony threshold is nearly immune to changes in $\alpha$ (as long as $\alpha$ is sufficiently large). This implies that a decoder employing phase-delayed inhibition has a synchrony threshold that is more robust to variations in the strength of excitation $\alpha$ than a decoder utilizing a high spike threshold; moreover, the synchrony threshold of the phase-delayed inhibition decoder is exceptionally robust to fluctuations in $\alpha$ if the strength of excitation $\alpha$ and the strength of inhibition $\beta$ vary together.

In a real neuronal system, if a read-out neuron employing phase-delayed inhibition receives input from $n$ encoders (so that each excitatory encoder input has strength $\frac{\alpha}{n}$ and each inhibitory interneuron input has amplitude $\frac{\beta}{n}$ ), it is likely that encoder activity is noisy, and so the number of the $n$ encoders that actually fire a spike will vary considerably from one oscillation cycle to the next. The net excitatory and inhibitory input amplitude will therefore vary from period to period, but they will vary together (i.e., if $k$ encoders fire during a particular oscillation cycle, the read-out neuron will receive a net excitation of $\frac{k \alpha}{n}$ and a net inhibition of $\frac{k \beta}{n}$ ). Since the synchrony threshold is relatively invariant to changes in $n$, this is effectively equivalent to $\alpha$ and $\beta$ varying from period to period with the ratio $\frac{\alpha}{\beta}$ remaining constant. Thus, it is likely that figure $7 \mathrm{~B}$ is most relevant to the robustness required of a real neuronal synchrony filter utilizing phase-delayed inhibition, and figure 7B shows that in the case that the ratio $\frac{\alpha}{\beta}$ is held fixed phase-delayed inhibition creates a synchrony threshold that is incredibly robust to fluctuations in encoder activity.

\subsubsection{Phase-Delayed Inhibition vs High Spike Threshold}

The analytical results of the previous section imply that a read-out neuron employing phase-delayed inhibition is capable of robustly detecting encoder syn- 


\section{Critical Excitation Required for Spiking \\ A}

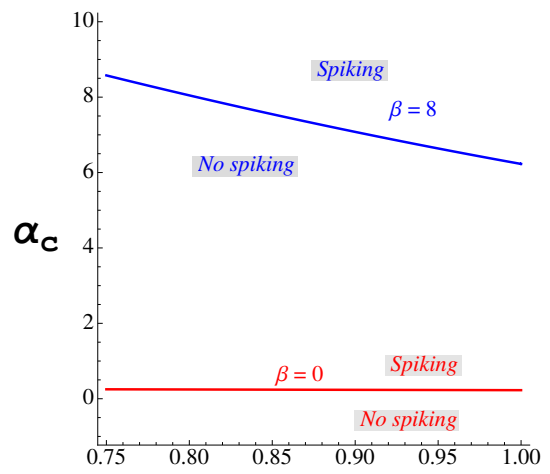

B

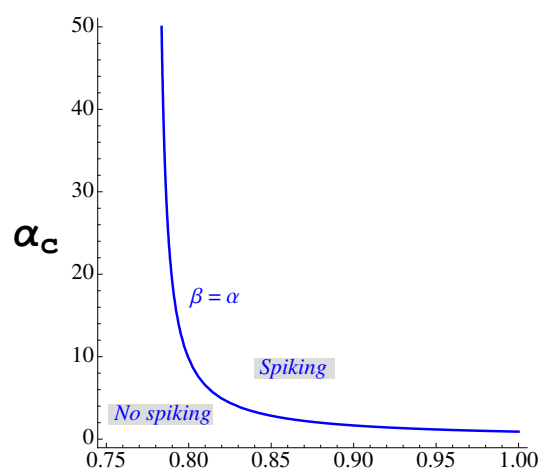

\section{synchrony parameter s}

Figure 7: Critical value of $\alpha\left(\alpha_{c}\right)$ required for the integrate-and-fire decoder to spike as a function of synchrony. A) The inhibitory amplitude was fixed at a value of $\beta=0$ or $\beta=8$. In the absence of inhibition, a synchrony threshold exists over a very narrow range of $\alpha$ values. With phase-delayed inhibition, a synchrony threshold exists over a broader range of $\alpha$ values. B) The inhibitory amplitude was varied with the excitatory amplitude $(\beta=\alpha)$. So long as $\alpha$ is sufficiently large, the synchrony threshold remains almost entirely untouched by changes in $\alpha$. Other parameters were fixed at $c=3 \mathrm{~ms}, h=5 \mathrm{~ms}, \omega=50$ $\mathrm{Hz}(T=20 \mathrm{~ms})$. chrony superimposed on a background of noisy encoder activity, while the synchrony detection properties of a read-out cell utilizing a high spike threshold are exquisitely sensitive to the background noise. Since it is well known that biological systems are inherently noisy, robustness to noise may provide the answer to the question of why the elaborate network architecture needed to implement phase-delayed inhibition would evolve in real neuronal systems. Below, we present computational results on the integrateand-fire decoder model that complement the analytical results of the previous section. We examine the robustness of the synchrony threshold in the case of a phase-delayed inhibition decoder versus in the case of a high spike threshold decoder in two key ways: 1) we fix the number of encoders that fire during an oscillation cycle and vary the strength of individual encoder and interneuron inputs; 2) we fix the strength of individual encoder and interneuron inputs and vary the number of encoders that fire during an oscillation cycle.

2.3.2.1 Input Strength: As shown in the analytically derived results of figure 7A, phase-delayed inhibition creates a synchrony threshold that is more resistant to small variations in the strength of excitation $\alpha$ than that created using a high spike threshold (where, with $n$ encoders, the strength of an excitatory input spike from an encoder is given by $\frac{\alpha}{n}$ ). In other words, if a read-out neuron employs phase-delayed inhibition (with a relatively low spike threshold), small changes in $\alpha$ (with the inhibitory amplitude $\beta$ held fixed) cause relatively small changes in the value of the synchrony threshold. If, on the other hand, a read-out neuron utilizes a relatively high spike threshold (without inhibition), then even slight changes in $\alpha$ entail substantial shifts in the value of the synchrony threshold. This analytically derived insight is supported by the computational results presented in figure $8 \mathrm{~A}$. In figure $8 \mathrm{~A}$, we vary the strength of excitatory encoder inputs $\alpha$ while fixing the amplitude of inhibitory interneuron inputs $\beta$ and fixing the number of encoders that fire during an oscillation cycle at $n$. Figure $8 \mathrm{~A}$ shows that phase-delayed inhibition creates a synchrony threshold that is more robust to 
Phase-Delayed Inhibition vs. High Spike Threshold

A) Robustness to strength of excitation
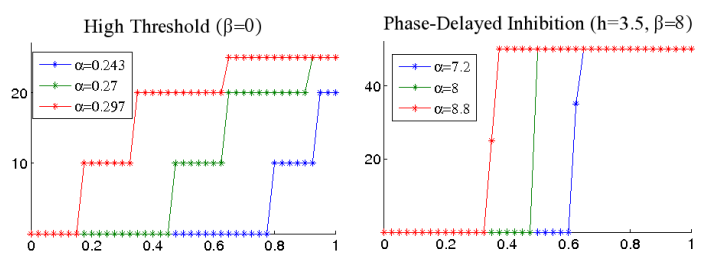

B) Phase Delayed Inhibition with fixed $\alpha / \beta$

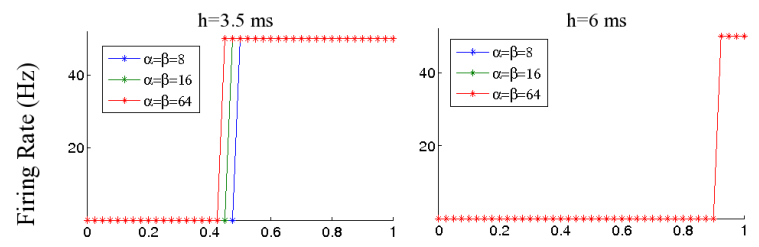

C) Robustness to input variability

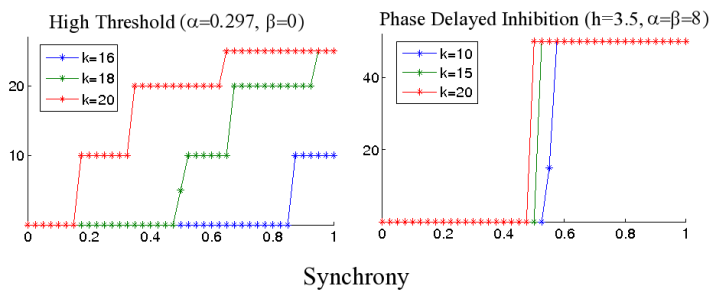

Figure 8: Robustness of synchrony filtering using phase-delayed inhibition vs high spike threshold within the integrate-and-fire decoder model. A) With a high spike threshold (and no inhibition), a 10\% change in the strength of total excitation $\alpha$ leads to large changes in the value of the synchrony threshold. If phase-delayed inhibition is employed, a 10\% change in $\alpha$ entails smaller changes in the value of the synchrony threshold. $B$ ) When using phase-delayed inhibition to create a synchrony filter, if the ratio of net excitatory to inhibitory strength $\left(\frac{\alpha}{\beta}\right)$ is fixed, then the value of the synchrony threshold is nearly impervious to changes in $\alpha$ (or $\beta$ ) and is determined almost entirely by the time course of inhibition $(h)$. In the right panel, the blue, green and red curves overlap completely and hence cannot be visually distinguished. $C$ ) Encoder number was fixed at $n=20$, with the amplitude of each individual encoder input given by $\frac{\alpha}{n}$ and the amplitude of each individual interneuron input given by $\frac{-\beta}{n}$. The parameters $\alpha, \beta, h, c$ were fixed in each panel, but rather than having all $n$ encoders spike during an oscillation cycle, we designated a number $k$ of the $n$ encoders to spike during the oscillation period. If a high spike threshold (without inhibition) is used to create a synchrony filter, small changes in $k$ lead to large variations in the value of the synchrony threshold. If phase-delayed inhibition is employed, then even large changes in $k$ lead to minimal deviations in the value of the synchrony threshold. Data shown are for an oscillation frequency of $\omega=50 \mathrm{~Hz}(T=20 \mathrm{~ms})$. 
changes in $\alpha$ than a high spike threshold decoder.

Moreover, we found that if $\alpha$ and $\beta$ are varied simultaneously, with the ratio $\frac{\alpha}{\beta}$ held fixed, then phasedelayed inhibition is even more robust - remarkably, the synchrony threshold remains untouched over a vast range of $\alpha$ and $\beta$ values. In fact, as demonstrated by the analytically derived results in figure $7 \mathrm{~B}$, the synchrony threshold rapidly approaches an asymptotic value as $\alpha$ and $\beta$ rise with the ratio $\frac{\alpha}{\beta}$ kept constant. Figure $8 \mathrm{~B}$ provides a computational complement to the analytic approach. In figure $8 \mathrm{~B}$, we simultaneously vary the strength of excitation $\alpha$ and the amplitude of inhibition $\beta$ with the ratio of the two held fixed at $\frac{\alpha}{\beta}=1$. Figure $8 \mathrm{~B}$ shows that phasedelayed inhibition creates a synchrony threshold that is staggeringly robust to changes in $\alpha$ when $\alpha$ and $\beta$ co-vary. This behavior occurs because a fixed value of $\frac{\alpha}{\beta}$ implies that the ratio of the time interval over which excitatory inputs decay to the time interval over which inhibitory inputs decay remains constant (if $\frac{\alpha}{\beta}=1$, then excitatory and inhibitory inputs decay over similar time intervals). Thus, with $\frac{\alpha}{\beta}$ fixed, the length of inhibitory input currents $h$ is the sole determinant of the relative time course of inhibition and excitation, implying that the value of the synchrony threshold depends on $h$ alone. This is assuming, as we have, that the length of excitatory input currents $c$ is held constant (it is really the ratio $\frac{h}{c}$ that determines the synchrony threshold). Note that this result implies that the more realistic integrate-and-fire decoder behaves in a virtually identical manner to our simplified decoder model given by a numerical threshold $\Theta$ imposed on the sum of step function inputs; fixing the ratio $\frac{\alpha}{\beta}$ effectively disentangles the amplitude from the time course of synaptic modulations of the integrate-and-fire decoder, and, as seen with the simplified decoder model, the synchrony threshold in the integrate-and-fire decoder is seen to exhibit a critical dependence on the time course of inputs alone (with the amplitude having little impact).

2.3.2.2 Input Number: Phase-delayed inhibition (with a low decoder spike threshold) allows for the creation of a dynamic synchrony filter capable of adapting to fluctuating input, while impos- ing a high spike threshold on the decoder (without phase-delayed inhibition) creates a stringent filter that is unable to dynamically accomodate variations in presynaptic activity. If a read-out cell receives input from $n$ encoders, then, in a noisy neuronal system, the actual number of encoder spikes may vary considerably from one oscillation cycle to the next. A high spike threshold implies that a certain number, say $m$, of those $n$ encoders must fire concurrently in order to drive the decoder. This number $m$ is invariant - regardless of how many of the $n$ encoders fire during an oscillation period, at least $m$ of these encoders must spike coherently to activate the read-out neuron. The level of synchrony (i.e., the fraction of input spikes that are coincident) required to drive the decoder therefore depends on the total number of encoders that fire during any given oscillation period. If a relatively small number of encoders fire during a given oscillation cycle, then a high proportion of them must be coincident to cause the decoder to spike, while if a relatively large number of encoders fire during a given oscillation cycle, a smaller fraction of them must be synchronous in order to activate the decoder. Phase-delayed inhibition, on the other hand, creates a read-out cell with a bona-fide synchrony threshold: irrespective of the exact number of encoder spikes during any particular oscillation period, a fixed fraction of those input spikes must temporally coincide in order to trigger the decoder. In figure $8 \mathrm{C}$, we fix $\alpha, \beta$, and $n$ (so that the strength of each excitatory encoder input is fixed at $\frac{\alpha}{n}$ and the amplitude of each inhibitory interneuron input is fixed at $\frac{\beta}{n}$ ), and we vary the number $k$ of the $n$ encoders that fire during an oscillation cycle. Figure $8 \mathrm{C}$ shows (computationally) that the synchrony threshold of the high spike threshold decoder is highly sensitive to variations in $k$, while the synchrony threshold of the phase-delayed inhibition decoder exhibits minimal shifts with even large changes in $k$. 


\subsection{Synchrony Filtering in Noisy Net- works}

In the above investigation on the integrate-and-fire decoder model, we made several biologically unrealistic assumptions in order to simplify analysis, allowing us to provide intuitively transparent results on the synchrony filtering properties of phase-delayed inhibition versus a high spike threshold. However, it is important to verify that the results obtained under these simplifying assumptions are applicable in a more biologically realistic setting. In this section, we obtain results using the integrate-and-fire decoder model with encoder and inhibitory interneuron inputs contructed in a more biologically realistic manner.

In the above results, encoder spike phases were deterministically distributed throughout a $T$ ms period, with increasing synchrony simulated by distributing spike phases evenly throughout smaller and smaller subintervals of the $T$ ms period. Biological networks, however, are inherently noisy, and hence in this section we distribute encoder spike phases in a stochastic manner. We simulate a synchrony level of $s=0$ by drawing encoder spike phases from a Gaussian distrubtion centered at the midpoint of a $T$ ms period with a standard deviation of $T$ ms; as the level of synchrony is increased from $s=0$ to $s=1$, the standard deviation of the Gaussian is decreased linearly from $T$ to $0 \mathrm{~ms}$. Moreover, in the above results we simulated encoder and inhibitory interneuron spikes as step function inputs to the integrate-and-fire decoder, with $\alpha, \beta$ denoting the amplitude and $c, h$ denoting the time course of the encoder and inhibitory interneuron input step functions, respectively. A more realistic description of synaptic inputs is given by a step function jump followed by exponential decay (Roth and van Rossum, 2009). In this section, we therefore simulate synaptic inputs to the integrate-and-fire decoder in a more realistic manner, with $\alpha, \beta$ denoting the size of the step function jump and $c, h$ denoting the time scale of exponential decay of encoder and inhibitory interneuron inputs, respectively. Additionally, for further realism we incorporate stochasticity into the synaptic delay $d$ between the arrival times of encoder and inhibitory interneuron inputs by draw-

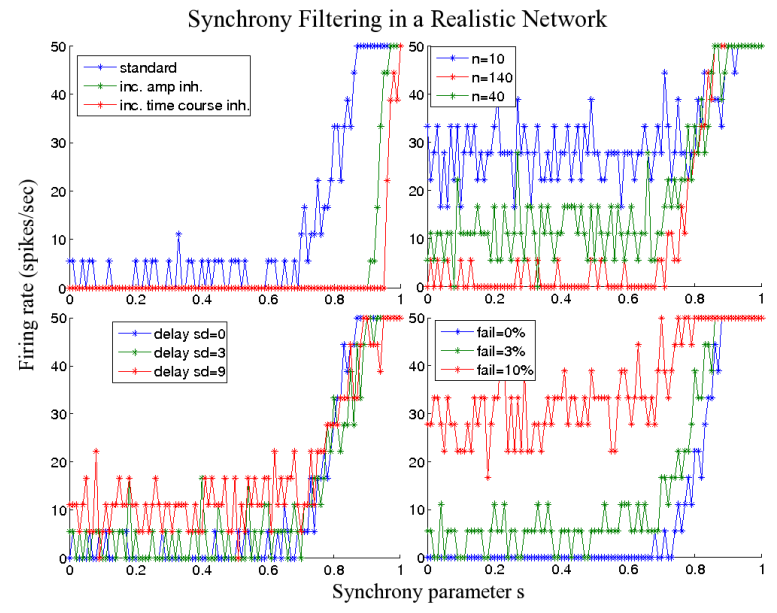

Figure 9: Synchrony filtering using an integrate-andfire decoder in a noisy, biologically realistic network (see text for model details). Similar to the deterministic case, the decoder possesses a sharp synchrony threshold, with the value of the synchrony threshold increasing with increases in the amplitude ( $\beta=4$ to $\beta=5)$ or time course $(h=3$ to $h=4)$ of inhibition (top left). Synchrony filtering is shown for varying encoder numbers $n$ (top right) and for varying degrees of stochasticity in the synaptic delay (bottom left; synaptic delays are drawn from a Gaussian distribution with mean $3 \mathrm{~ms}$ and different standard deviations), and a varying probability of synaptic failure (bottom right; failure probability shown as a percentage). Data shown are for an oscillation frequency of $\omega=50 \mathrm{~Hz}(T=20 \mathrm{~ms})$.

ing $d$ from a Gaussian distrubution with mean $3 \mathrm{~ms}$ and standard deviation denoted by delaysd. Finally, since in a realistic setting each encoder spike may not elicit an inhibitory interneuron spike, we set a probability of synaptic failure of inhibitory interneuron spikes, with the parameter fail (written as a percentage) denoting the chance that an encoder spike does not elicit a corresponding inhibitory interneuron spike.

In the simulations presented in this section, standard values of the network parameters mentioned 
above are given by the following: $\alpha=\beta=4$, $c=h=3 \mathrm{~ms}$, delaysd $=1 \mathrm{~ms}$, fail $=2 \%$. The standard number of encoders is set at $n=100$ and the oscillation period is set at $T=20 \mathrm{~ms}$. Unless otherwise stated, all simulations are performed with these standard parameter values.

In figure 9, we plot simulation results on the synchrony filtering properties of an integrate-and-fire decoder employing phase-delayed inhibition in this more biologically realistic setting. Figure 9 (top left) shows that, while synchrony response curves are noisy, the decoder continues to exhibit sharp synchrony filtering properties, with increases in the amplitude $\beta$ or decay time scale $h$ of inhibition causing a rightward shift in the value of the synchrony threshold. However, as shown in the top right panel, a larger number $n$ of encoder neurons and inhibitory interneurons is required to achieve a sharp synchrony threshold than in the simplified model presented in the previous section (in the biologically realistic setting, a minimum of $\sim n=40$ is needed, while in the simplified model of the previous section a minimum of $\sim n=10$ is required). This discrepancy can be explained by the stochastic nature of encoder spike phases in the biologically realistic setting - a larger number of cells $n$ is required in order for the decoder to be able to respond to the mean behavior of arrival times of encoder and inhibitory interneuron spikes. In the bottom left panel, we show that significant variations in delaysd have little effect on the synchrony filtering properties of the decoder, implying that while a synaptic delay between excitatory and inhibitory inputs is needed, the exact value of the synaptic delay has little bearing on the response properties of the decoder. In the bottom right panel, we show that increasing the synaptic failure parameter fail reduces the sharpness of the synchrony threshold - this occurs because a higher level of synaptic failure diminishes the net inhibition impinging upon the deocder, mitigating the efficacy of the phase-delayed inhibition architecture.

In figure 10, we compare the synchrony filtering properties of a decoder employing phase-delayed inhibition versus a decoder utilizing a high spike threshold in a biologically realistic setting. In the left panel, we show that a decoder employing phase-delayed in- hibition exhibits incredibly robust synchrony filtering properties in the case that excitation and inhibition are balanced (i.e., if the ratio $\frac{\alpha}{\beta}$ is held fixed). This is consistent with the results from the previous section, implying that phase-delayed inhibition (in which network architecture automatically creates balanced levels of excitation and inhibition delivered to the decoder) allows for strikingly sharp and robust synchrony detection. In the right panel, we show that a decoder employing a high spike threshold displays no such robustness; even after fine-tuning $\alpha$ to create as sharp a synchrony detector as possible, we find that the synchrony threshold is not as sharp as in the phase-delayed inhibition case and that the effectiveness of synchrony filtering is dramatically diminished by small variations in $\alpha$.

Phase-Delayed Inhibition vs High Spike Threshold

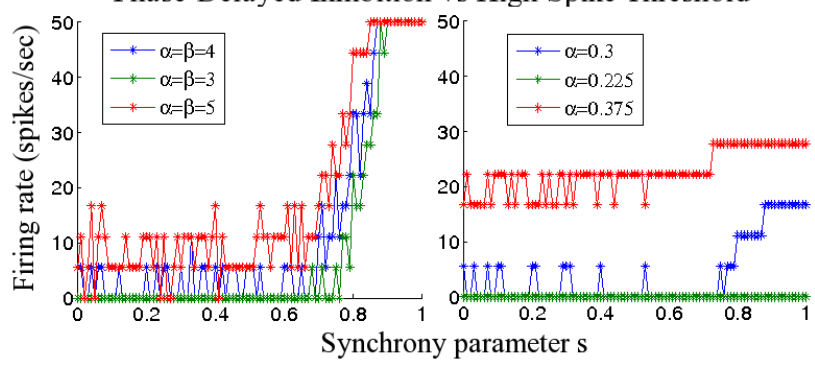

Figure 10: Phase-delayed inhibition versus a high spike threshold using an integrate-and-fire decoder in a noisy, biologically realistic network (see text for model details). Left: Synchrony filtering with phasedelayed inhibition. As in the deterministic case, if the ratio $\frac{\alpha}{\beta}$ is held fixed (i.e., if the decoder receives excitation and inhibition in fixed proportion), phase-delayed inhibition creates an exceptionally robust synchrony threshold ( $\alpha$ and $\beta$ are varied from baseline by $25 \%$ in the panel). Right: Synchrony filtering with a high spike threshold. Varying the amplitude of excitation $\alpha$ by $25 \%$ causes drastic changes in the synchrony filtering properties of the decoder. Data shown are for an oscillation frequency of $\omega=50$ $\mathrm{Hz}(T=20 \mathrm{~ms})$. 


\section{Discussion}

Neuronal populations within a diverse array of brain regions encode and transmit information through synchronized oscillations, implying that a mechanism must exist that is capable of decoding the coherent, periodic activity of a group of neurons. Two realistic mechanisms exist for designing such a decoder neuron: 1) imposing a relatively high spike threshold on the decoder, or 2) employing a phase-delayed inhibition network motif. The manner in which a high spike threshold can create a decoder neuron that acts as a coincidence detector is obvious. In this work, we have shown that phase-delayed inhibition can also enable a decoder to act as a high-pass synchrony filter, in the sense that below a threshold level of input synchrony, the spiking response of the read-out cell is virtually nonexistent, but as soon as the inputs exceed this synchrony threshold the decoder's response jumps to high values. Moreover, we have demonstrated that the the time course of inhibition plays a pivotal role in determinining the synchrony threshold.

Furthermore, we have shown that phase-delayed inhibition provides for more robust synchrony filtering than a high spike threshold, explaining its utilization despite the neccesity for a more intricate network architecture. Phase-delayed inhibition was found to be more robust to fluctuations in both input strength and input number than a high spike threshold. Robustness to input number is a particularly important quality, since a decoder of synchronized oscillations in a real neuronal system is likely to receive a variable number of encoder spikes from one oscillation period to the next. With phase-delayed inhibition, the decoder requires a particular minimum level of synchrony among its inputs (i.e., a fixed fraction of input spikes must temporally overlap), regardless of the exact number of encoder spikes impinging upon the decoder. In contrast, a high spike threshold creates a decoder whose synchrony filtering properties vary drastically with fluctuations in the number of encoder spikes.

Thus, phase-delayed inhibition can be viewed as adaptively regulating the effective spike threshold of the decoder to filter out background noise. In the presence of a small amount of background encoder noise, phase-delayed inhibition sets the spike threshold of the decoder at a relatively low value (i.e., a small number of synchronous encoder spikes must occur in order to activate the decoder). In the presence of a large amount of background encoder noise, phase-delayed inhibition sets the spike threshold of the decoder at a relatively high value (i.e., a large number of coincident encoder spikes are required in order to trigger the decoder). This type of adaptive regulation of the read-out cells's spike threshold can also be attained by using a high spike threshold decoder (without inhibition) to detect synchrony, provided that the strength of excitatory synapses is plastic; if the strength of excitatory synapses varies inversely with the net amount of encoder activity, a high spike threshold decoder may be able to perform the same computations as a phase-delayed inhibition decoder. The likely advantage of using phase-delayed inhibition, rather than a high spike threshold decoder with plastic synapses, is that phase-delayed inhibition allows essentially instantaneous regulation of the effective spike threshold of the decoder, while synaptic plasticity requires time in order to respond to a particular level of net encoder activity. In systems that exhibit synchronized oscillations, encoder activity may vary considerably from one oscillation cycle to the next, and so a mechanism that instantaneously tracks changing levels of net encoder activity (such as phase-delayed inhibition) would be far better than a mechanism which tracks changing levels of net encoder activity more slowly (such as a high spike threshold decoder with synaptic plasticity).

\subsection{Decoder Models}

In our investigations, we used both a simplified model of decoder activity (a threshold $\Theta$ imposed on the sum of the step function inputs) as well as a more realistic integrate-and-fire read-out neuron model. Perhaps surprisingly, the results obtained using the simplfiied model were highly concordant with those produced by the more realistic model, suggesting that, for certain purposes, step function inputs along with an arbitrarily imposed threshold may provide an acccessible yet accurate approximation to neuronal activity. One essential advantage of the simplified 
model is the ability to cleanly tease apart the amplitude from the time course of an input, which allowed us to generate clear-cut results on the individual effects of $\beta$ or $h$. With an integrate-and-fire decoder, however, the time course and magnitude of an input are inextricably linked; since the membrane potential $V(t)$ exhibits non-instantaneous dynamics, large inputs take longer to decay than smaller inputs. Thus, the consequences of varying $\beta$ versus those of changing $h$ are intermingled (compare figures 3 and 6 ).

\subsection{Other Modeling Work}

There is a vast body of prior work on balanced networks (i.e., networks in which neurons receive proportional amounts of excitation and inhibition). In a balanced network, the mean level of inhibition impinging upon a neuron tends to cancel the mean level of incoming excitation - a neuron therefore spikes sporadically and irregularly, when an instantaneous fluctuation in the net inhibitory and/or net excitatory current to the cell allows its membrane potential to climb to threshold (e.g., see Shadlen and Newsome, 1994; Troyer and Miller, 1997; Amit and Brunel, 1997; van Vreeswijk and Sompolinsky, 1996; Brunel, 2000; Kumar et al., 2008, Vogels and Abbott, 2009). The principle of balanced excitation and inhibition applies to the phase-delayed inhibition architecture. The decoder neuron within a phase-delayed inhibition scheme receives proportional amounts of excitation and inhibition, with the mean level of inhibitory interneuron input canceling the mean level of excitatory encoder input, and the decoder fires only if encoder synchrony causes large moment-to-moment fluctuations in the net amount of excitation and inhibition to the decoder.

Burck and van Hemmen (2009) studied phasedelayed inhibition using a mathematical approach. These authors modeled a population of input and output neurons as inhomogeneous Poisson processes, with spikes of the input population feeding both excitation and temporally lagging inhibition to the output population. The activity of the input population was oscillatory, and the authors studied the dependence of the preferred frequency of the output population on the time constant of excitation, the time constant of inhibition, and the delay between excitation and inhibition. Kremkow et al. (2010a,2010b) constructed a computational neuronal network model to show that the presence of inhibition staggered in time can suppress the response of a read-out neuron to asynchronous inputs, as well as sharpen the temporal precision of read-out cell spikes. Moreover, the authors show that embedding time-delayed feedforward inhibition within a synfire chain allows selective propagation of synchronous inputs, and further that embedding the phase-delayed inhibition architecture within a larger network provides a method for gating of incoming signals. Akam and Kullmann (2010) also adopted a computational approach, and showed that a network of inhibitory interneurons that supply feedforward inhibition with a temporal delay can allow selective transmission of a synchronized, oscillating input (within a particular frequency band) in the presence of several asynchronous distractor stimuli. Assisi et al. (2007) showed that a circuit utilizing phase-delayed inhibition can maintain sparse read-out cell activity over a broad range of stimulus intensities (see below). The authors of the present paper have shown in other work that stimuli encoded by synchronous activity of a neuronal population can be decoded in both a reliable and stimulus-specific manner by a phase-delayed inhibition architecture, but not by a decoder employing a high spike threshold (Joshi and Patel, 2013). However, these models, while examining properties of phase-delayed inhibition and exploring its possible physiological roles, do not address the fundamental computation performed by the phase-delayed inhibition architecture. In this paper, we frame the problem of phase-delayed inhibition in its most mathematically simplified form, allowing us to precisely delineate the nature of the computation performed by a synchrony detection mechanism employing phase-delayed inhibition.

\subsection{Real Neural Systems}

The phase-delayed inhibition motif likely exists in a variety of neural systems as a means to decode synchronized oscillations; two representative examples include the locust olfactory system and the barn owl optic tectum. The locust antennal lobe (AL) receives 
direct input from olfactory receptor cells and exhibits a $20 \mathrm{~Hz}$ network oscillation in response to odor stimulation, with odor features modulating the identity of active neurons but having no effect on oscillation frequency (Laurent and Naraghi, 1994; Mazor and Laurent, 2005; Wehr and Laurent, 1996). Approximately 800 excitatory projection neurons (PNs) within the AL project to a large array of $\sim 50,000$ Kenyon cells (KCs), with each $\mathrm{KC}$ reading from $\sim 400 \mathrm{PNs}$ (Jortner et al., 2007). PN axons also innervate the lateral horn, a structure consisting of GABAergic interneurons which may supply vigorous, phase-delayed inhibition to the KC decoders (Leitch and Laurent, 1996; Perez-Orive et al., 2002). Prior modeling work has shown that this feedforward inhibitory circuit may contribute to $\mathrm{KC}$ coincidence detection properties and in maintaining sparse $\mathrm{KC}$ odor representations over a broad range of odorant concentrations (Assisi et al., 2007; Perez-Orive et al., 2004). This result is encompassed by the more general investigation of phase-delayed inhibition carried out in this paper; our results show that decoder activity jumps and rapidly saturates once the inputs exceed a certain threshold level of synchrony, and since increasing odorant concentration increases PN synchrony within the locust $\mathrm{AL}$, our results imply that $\mathrm{KC}$ responses will remain invariant to changes in odor concentration (so long as PN synchrony exceeds a threshold value). Examination of $\mathrm{KC}$ odor representations in light of the principles of phase-delayed inhibition presented in this paper may yield further insights into this system.

Within the deep layers of the barn owl optic tectum (OT), cells respond to both visual and auditory stimuli and are topographically organized, with adjacent cells displaying neighboring spatial receptive fields (Knudsen, 1982; Knudsen, 1983; Knudsen and Brainard, 1991; Knudsen and Knudsen, 1983). Auditory and visual stimuli within a restricted region of space elicit coordinated, $25-90 \mathrm{~Hz}$ gamma oscillations in cells located at the corresponding location within the topographically organized OT. Interestingly, the spatial tuning curve and contrast-response function of an OT site vary depending on whether responses are measured in terms of firing rate or gamma power, suggesting that spike rate and gamma oscillations may be used to relay information in paral- lel (Patel and Reed, 2013; Sridharan et al., 2011). Tectal fibers stream towards the nucleus rotundus of the thalamus, emanating collaterals en-route to innervate the GABAergic pretectal nuclei, whose axons course towards rotundal cells as well (Benowitz and Karten, 1976; Deng and Rogers, 1998; Karten et al., 1997; Karten and Revzin, 1966; Luksch et al., 1998; Mpodozis et al., 1996; Tombol et al., 1994). Neurons of the nucleus rotundus are therefore in an ideal position to use phase-delayed inhibition to selectively decode the synchronized gamma oscillations of their tectal inputs (Patel and Reed, 2013).

In addition providing a method for deciphering periodic network behavior, phase-delayed inhibition has also been shown to assist in the decoding of population activity that is not necessarily oscillatory. In these cases, phase-delayed inhibition may still play a similar role in allowing a read-out cell to act as a coincidence detector or shortening the temporal window over which a read-out neuron can integrate excitatory inputs. Hippocampal pyramidal neurons have been shown to receive direct excitation via Schaffer collaterals as well as disynaptic inhibition from the same source with a time delay of $\sim 2 \mathrm{~ms}$, causing these cells to behave as coincidence detectors (Fricker and Miles, 2000; Pouille and Scanziani, 2001). Within the auditory cortex, investigators have demonstrated that excitatory and inhibitory inputs to cortical cells have similar frequency and intensity tuning; inhibitory inputs, however, arrive with a 1-4 ms delay relative to excitatory inputs, sharpening the temporal precision of cortical cell spikes (Wehr and Zador, 2003). The cerebellum is thought to play a crucial role in the coordination of movements over millisecond time scales, and to that end cerebellar Purkinje cells are thought to act as precise coincidence detectors within a circuit employing phase-delayed inhibition. Parallel fibers produce direct excitation, as well as indirect inhibition via molecular layer interneurons, within Purkinje cells, with excitation preceding inhibition by 1-2 ms (Mittmann et al., 2005). Phase-delayed inhibition has also been observed within the lateral geniculate nucleus (LGN) of the thalamus. Within LGN neurons, IPSPs have been recorded that are locked to EPSPs induced by retinal ganglion cells (RGC), in the sense that each EPSP is followed with a 1 
ms time lag by an IPSP; furthermore, these locked IPSPs have been shown to sharply curtail the time window over which LGN neurons can respond to excitation, enhancing the temporal precision of LGN spiking (Blitz and Regehr, 2005).

In this paper, we studied phase-delayed inhibition as a method for decoding synchronized oscillations, and we therefore derived results in the steady-state case (i.e., we discarded the first few oscillation cycles in order to disregard the initial transient behavior of the decoder). In systems that utilize phase-delayed inhibition without oscillations, the effects of inhibition are likely to be better described by the initial transient behavior of the decoder (i.e., by the decoder response within the first oscillation cycle). In preliminary investigations, we have seen that similar general principles to those presented in this paper may govern the initial transient behavior of the decoder.

Finally, it would be a relatively simple matter to experimentally verify the results presented in this paper. Using an in vitro preparation, an investigator could simulate encoder and interneuron inputs by injecting excitatory and inhibitory current pulses into a single neuron. By appropriately modulating the timing and amplitude of the current pulses, the spiking activity of the neuron would then be indicative of the response of a decoder of phase-delayed inhibition.

\section{Methods}

The construction of step function inputs, and the simplified decoder model in which we impose a numerical threshold $\Theta$ on the step function sum, are described in full detail in the Model Construction section of the Results. The integrate-and-fire decoder model was governed by the following equation:

$$
\frac{d V}{d t}=-g V+i(t)
$$

where $V(t)$ is the membrane potential, $g$ is the leak conductance, and $V_{0}=0$ is the resting potential. The model is nondimensionalized, with $g=0.05 \mathrm{~ms}^{-1}$, a nondimensional membrane potential, time in units of $\mathrm{ms}$, and the synaptic input $i(t)$ having units of $\mathrm{ms}^{-1}$. A spike was recorded when $V(t)$ reached a threshold value $V_{\text {thresh }}=1$, with $V(t)$ being instantaneously reset to rest following a spike. An absolute refractory period of $2 \mathrm{~ms}$ was simulated by holding the membrane potential at rest for $2 \mathrm{~ms}$ following a spike. Simulations were also performed with no refractory period and a refractory period of $1 \mathrm{~ms}$, which yielded similar results. Details of the nondimensional model are given in Tao et al. (2004).

We chose an integrate-and-fire model because we were primarily concerned with the number of spikes generated, rather than the precise timing of individual spikes, and hence detailed modeling of a spike generation mechanism was unnecessary. Additionally, we modeled synaptic inputs as current-based, rather than conductance-based, in order to simplify the mathematical analysis that we carry out in the Results section. All simulations were also performed using conductance-based inputs, which yielded similar results.

The synaptic input $i(t)$ was described as the sum of the excitatory and inhibitory step function inputs to the neuron, as described in the Results. Spike rates were measured by averaging over $\sim 5$ oscillation cycles, after discarding the first several oscillation cycles in order to ignore the initial transient and to measure only steady-state responses. Simulations were carried out using the explicit Euler method with a time step of $0.001 \mathrm{~ms}$. The small time step required for convergence of the numerical method was a consequence of the discontinuous synaptic inputs.

\section{Acknowledgements}

Mainak Patel was supported by a National Science Foundation grant (DMS-0943760). Badal Joshi was partially supported by a National Science Foundation grant (EF-1038593).

\section{References}

Akam T and Kullmann D. (2010). Oscillations and filtering networks support flexible routing of information. Neuron. 67, 308-320.

Amit D and Brunel N. (1997). Model of global spontaneous activity and local structured activity during delay periods in 
the cerebral cortex. Cereb Cortex. 7, 237-252.

Assisi C, Stopfer M, Laurent G, and Bazhenov M. (2007). Adaptive regulation of sparseness by feedforward inhibition. Nat Neurosci. 10, 1176-1184.

Benowitz L and Karten H. (1976). Organization of the tectofugal visual pathway in the pigeon: a retrograde transport study. J Comp Neurol. 167, 503-520.

Blitz D and Regehr W. (2005). Timing and specificity of feedforward inhibition within the LGN. Neuron. 45, 917-928.

Brunel N. (2000). Dynamics of networks of randomly connected excitatory and inhibitory spiking neurons. J Physio (Paris). 94, 445-463.

Burck M and van Hemmen J. (2009). Neuronal identification of signal periodicity by balanced inhibition. Biolog Cybernet. $100,261-270$.

Deng C and Rogers L. (1998). Organisation of the tectorotundal and SP/IPS-rotundal projections in the chick. J Comp Neurol. 394, 171-185.

Eckhorn R. (1994). Oscillatory and non-oscillatory synchronizations in the visual cortex and their possible roles in associations of visual features. Prog Brain Res. 102, 405-426.

Fricker D and Miles R. (2000). EPSP amplification and the precision of spike timing in hippocampal neurons. Neuron. 28, 559-569.

Friedrich R, Habermann C, and Laurent G. (2004). Multiplexing using synchrony in the zebrafish olfactory bulb. Nat Neurosci. 7, 862-871.

Gray C. (1994). Synchronous oscillations in neuronal systems: mechanisms and functions. J Comput Neurosci. 1, 11-38.

Jortner R, Farivar S, and Laurent G. (2007). A simple connectivity scheme for sparse coding in an olfactory system. J Neurosci. 27, 1659-1669.

Joshi B and Patel M. (2013). Encoding with synchrony: phasedelayed inhibition allows for reliable and specific stimulus detection. J Theor Biol. 328, 26-32.

Karten H, Cox K, and Mpodozis J. (1997). Two distinct populations of tectal neurons have unique connections within the retinotectorotundal pathway of the pigeon (Columba livia). J Comp Neurol. 387, 449-465.

Karten H and Revzin A. (1966). The afferent connections of the nucleus rotundus in the pigeon. Brain Res. 2, 368-377.

Kleppe I and Robinson H. (1999). Determining the activation time course of synaptic AMPA receptors from openings of colocalized NMDA receptors. Biophys J. 77, 1418-1427.

Knudsen E. (1982). Auditory and visual maps of space in the optic tectum of the owl. J Neurosci. 2, 1177-1194.

Knudsen E. (1983). Early auditory experience aligns the auditory map of space in the optic tectum of the barn owl. Science. 222, 939-942.
Knudsen E and Brainard M. (1991). Visual instruction of the neural map of auditory space in the developing optic tectum. Science. 253, 85-87.

Knudsen E and Knudsen P. (1983). Space-mapped auditory projections from the inferior colliculus to the optic tectum in the barn owl (Tyoto alba). J Comp Neurol. 218, 187-196.

Kremkow J, Aertsen A, and Kumar A. (2010a). Gating of signal propagation in spiking neural networks by balanced and correlated excitation and inhibition. J Neurosci. 30, 1576015768 .

Kremkow J, Perrinet L, Masson G, and Aertsen A. (2010b). Functional consequences of correlated excitatory and inhibitory conductances in cortical networks. J Comput Neurosci. 28, 579-594.

Kumar A, Schrader S, Aertsen A, and Rotter S. (2008). The high conductance state of cortical networks. Neural Comput. 20, 1-43.

Laurent G and Davidowitz H. (1994). Encoding of olfactory information with oscillating neural assemblies. Science. 265, $1872-1875$.

Laurent G and Naraghi M. (1994). Odorant-induced oscillations in the mushroom bodies of the locust. J Neurosci. 14, 2993-3004.

Leitch B and Laurent G. (1996). GABAergic synapses in the antennal lobe and mushroom body of the locust olfactory system. J Compar Neurol. 372, 487-514.

Loring R and Zigmond R. (1988). Characterization of neuronal nicotinic receptors by snake venom neurotoxins. Trends Neurosci. 11, 73-78.

Luksch H, Cox K, and Karten H. (1998). Bottlebrush dendritic endings and large dendritic fields: motion-detecting neurons in the tectofugal pathway. J Comp Neurol. 396, 399-414.

Marthy V and Fetz E. (1992). Coherent 25- to 35-Hz oscillations in the sensorimotor cortex of awake behaving monkeys. Proc Natl Acad Sci USA. 89, 5670-5674.

Mazor O and Laurent G. (2005). Transient dynamics versus fixed points in odor representations by locust antennal lobe projection neurons. Neuron. 48, 661-673.

Mittmann W, Koch U, and Hausser M. (2005). Feed-forward inhibition shapes the spike output of cerebellar Purkinje cells. J Physiol. 563, 369-378.

Mpodozis J, Cox K, Shimizu T, Bischof H, Woodson W, and Karten H. (1996). GABAergic inputs to the nucleus rotundus (pulvinar inferior) of the pigeon (Columba livia). J Comp Neurol. 374, 204-222

Oertel D, Bal R, Gardner S, Smith P, and Joris P. (2000). Detection of synchrony in the activity of auditory nerve fibers by octopus cells of the mammalian cochlear nucleus. PNAS. 97, 11773-11779.

Patel M and Reed M. (2013). Stimulus encoding within the barn owl optic tectum using gamma oscillations vs. spike rate: 
a modeling approach Network. Epub ahead of print.

netics. Brain Res. 780, 166-169.

Perez-Orive J, Bazhenov M, and Laurent G. (2004). Intrinsic and circuit properties favor coincidence detection for decoding oscillatory input. J Neurosci. 24, 6037-6047.

Perez-Orive J, Mazor O, Turner G, Cassenaer S, Wilson R, and Laurent G. (2002). Oscillations and sparsening of odor representations in the mushroom body. Science. 297, 359-365.

Pouille F and Scanziani M. (2001). Enforcement of temporal fidelity in pyramidal cells by somatic feed-forward inhibition. Science. 293, 1159-1163.

Roth A and van Rossum M. (2009). Modeling synapses. In Computational modeling methods for neuroscientists, E.D. Shutter, ed. (Cambridge, MA : MIT Press), pp. 139-160.

Shadlen M and Newsome W. (1994). Noise, neural codes and cortical organization. Curr Opin Neurobio. 4, 569-579.

Sridharan D, Boahen K, and Knudsen E. (2011). Space coding by gamma oscillations in the barn owl optic tectum. J Neurophysiol. 105, 2005-2017.

Steriade M. (2006). Grouping of brain rhythms in corticothalamic systems. Neuroscience. 137, 1087-1106.

Tao L, Shelley M, McLaughlin D, and Shapley R. (2004). An egalitarian network model for the emergence of simple and complex cells in visual cortex. Proc Natl Acad Sci. 101, 366371.

Thany S. (2010). Insect nicotinic acetylcholine receptors (Austin, Texas: Landes Bioscience and Springer Science).

Titz S and Keller B. (1997). Rapidly deactivating AMPA receptors determine excitatory synaptic transmission to interneurons in the nucleus tractus solitarius from rat. J Neurophysiol. 78, 82-91.

Tombol T, Egedi G, and Nemeth A. (1994). Phaseolus vulgaris lectin labelled and GABA immunogold stained terminals in nucleus rotundus. An EM study. J Hirnforsch. 35, 233-252.

Troyer T and Miller K. (1997). Physiological gain leads to high ISI variability in a simple model of a cortical regular spiking cell. Neural Comput. 9, 971-983.

van Vreeswijk C and Sompolinsky H. (1996). Chaos in neuronal networks with balanced excitatory and inhibitory activity. Science. 274, 1724-1726.

Vogels T and Abbott L. (2009). Gating multiple signals through detailed balance of excitation and inhibition in spiking networks. Nat Neurosci. 12, 483-491.

Wehr M and Laurent G. (1996). Odor encoding by temporal sequences of firing in oscillating neural assemblies. Nature. 384, 162-166.

Wehr M and Zador A. (2003). Balanced inhibition underlies tuning and sharpens spike timing in auditory cortex. Nature. $426,442-446$.

Zhou F and Hablitz J. (1998). AMPA receptor-mediated EP$\mathrm{SCs}$ in rat neocortical layer II/III interneurons have rapid ki- 


\section{Supplementary Material}

In this section we describe the analytic derivation of the synchrony threshold of the integrate-and-fire decoder. In particular, we derive the function $\alpha_{c}(s)$, where $\alpha_{c}$ is the critical value of $\alpha$ at which the decoder transitions from quiescence to spiking, and $s$ is the level of synchrony among the encoders.

Recall from Section 2.3 that $i(t)=\sum_{j=1}^{n} i_{j}(t)$ is the total input current from $n$ excitatory encoder neurons and $n$ inhibitory interneurons. The effective $j$-th input is the sum of the excitation from the $j$-th encoder and the inhibition from the corresponding $j$-th interneuron.

$$
i_{j}(t)= \begin{cases}\alpha / n, & \text { if } t(\bmod T) \in\left(\varphi_{j}, \varphi_{j}+c\right) \\ -\beta / n, & \text { if } t(\bmod T) \in\left(\varphi_{j}+c, \varphi_{j}+c+h\right) \\ 0, & \text { elsewhere }\end{cases}
$$

where $\alpha, \beta, c, h, T>0, c+h<T$ and $\varphi_{j}$ is the relative phase of the $j$-th neuron with $\varphi_{1}:=0$. For simplicity, we assume the phases to be uniformly distributed over a 'window' of size $w<T$, so that $\varphi_{j}=-(j-1) w / n$.

In the limit $n \rightarrow \infty$, assuming that the readout neuron voltage $V(t)$ remains below the spike threshold of $V_{\text {thresh }}=1$ for all time, the time course of the voltage of the readout neuron is governed by the following equations:

$$
\begin{gathered}
\dot{V}(t)=-g V(t)+i(t) \\
i(t)=\frac{1}{w} \int_{t}^{t+w} I(u) d u, \text { where } \\
I(t)= \begin{cases}\alpha, & \text { if } t(\bmod T) \in(0,+c) \\
-\beta, & \text { if } t(\bmod T) \in(c, c+h) \\
0, & \text { if } t(\bmod T) \in(c+h, T)\end{cases}
\end{gathered}
$$

1. Assuming that the parameters are such that $V(t)$ remains below $V_{\text {thresh }}=1$ for all time $t$, the system follows equations (1) and (2).

2. We are only interested in the steady-state behavior of the readout neuron, so we look for solutions of the differential equations which have the same periodic behavior as $i(t)$; in other words, the solution $V(t)$ has the property that $V(t+T)=V(t)$ for all time $t$. All initial conditions result in the solution converging to this periodic solution.

3. The condition that $V(t)<V_{\text {thresh }}$ is equivalent to the condition that the maximum value of the voltage $\left(V_{\max }\right)$ on a single period is less than the spiking threshold $V_{\text {thresh }}$. We impose this condition to obtain the relation between $\alpha$ and the other parameters to ensure that the readout neuron does not spike.

We now state the no spiking condition (refer to Theorem 1). When the parameters satisfy the no spiking inequality (corresponding to $V_{\max }<1$ ), the read-out neuron does not fire any spikes once the potential has converged to steady state behavior. In other words, the read-out neuron is quiescent (after the initial transient). On the other hand, if $V_{\max } \geq 1$, then the read-out neuron fires repetitively. In the following, we define the synchrony parameter $s:=1-w / T \in(0,1) . s=1$ indicates perfect synchrony (where all the inputs have the same phase) while $s=0$ indicates perfect discordance (where the phases are uniformly distributed over the entire period). 
Theorem 4.1 (No spiking condition). Let $s>1-h / T$. Let

$$
\bar{V}(\alpha, s):=\frac{\alpha}{g}-\frac{\alpha+\beta}{g^{2} T(1-s)} \ln \left[1+\frac{e^{g T(1-s)}-1}{e^{g T}-1}\left(\frac{\alpha\left(e^{g(T-c)}-1\right)+\beta\left(e^{g h}-1\right)}{\alpha+\beta}\right)\right]
$$

and let $\alpha_{c}(s)$ be the implicitly defined function whose graph is the curve $\bar{V}\left(\alpha_{c}, s\right)=1$. In fact, if $\alpha$ and $\beta$ co-vary then by defining $\kappa:=\beta / \alpha$ the critical excitation strength may be explicitly written as

$$
\begin{gathered}
\alpha_{c}(s)=\left(\frac{1}{g}-\frac{1+\kappa}{g^{2} T(1-s)} \ln \left[1+\frac{e^{g T(1-s)}-1}{e^{g T}-1}\left(\frac{\left(e^{g(T-c)}-1\right)+\kappa\left(e^{g h}-1\right)}{1+\kappa}\right)\right]\right)^{-1} \\
\text { If } \alpha\left\{\begin{array}{l}
<\alpha_{c}(s) \\
\geq \alpha_{c}(s) \text { the readout neuron does not spike }
\end{array}\right.
\end{gathered}
$$

We will first state and prove an algorithm for deriving the condition on the input current $i(t)$ such that the readout neuron is in quiescent mode. A particular application of the algorithm results in a proof of the Theorem (no spiking condition) stated above. We will apply the algorithm to the two special cases where $0<w \leq c$ and $c<w \leq h$.

Nondimensionalizing time $t$ allows us to eliminate the conductance parameter $g$. We use a tilde $(\sim)$ to indicate the nondimensional variables and parameters. In terms of the original dimensional quantities we have $\tilde{t}=g t, \tilde{w}=g w, \tilde{T}=g T, \tilde{c}=g c, \tilde{h}=g h, \tilde{\alpha}=\alpha / g, \tilde{\beta}=\beta / g$. Let $\tilde{i}(\tilde{t})$ be the current in terms of the nondimensional variables. The fully nondimensional leaky integrate and fire model is

$$
\frac{d V}{d \tilde{t}}=-V+\tilde{i}(\tilde{t})
$$

with a reset to $V\left(\tilde{t}^{+}\right)=V_{0}$ when $V(\tilde{t})=V_{\text {thresh }}>V_{0}$. For convenience, we will drop the tilde from now on. We now state the above-mentioned algorithm.

Algorithm (Output: No spiking condition) The algorithm produces a condition on parameter values such that the neuron is quiescent, after a possible initial transient. Fix $w>0$. Let $\bar{t}$ be such that $V(\bar{t}) \geq V(t)$ for all $t$. Let $p$ be an interval containing $\bar{t}$ where $i(t)$ is continuously differentiable and let $t_{0}$ be the left end-point of $p$.

Step 1. Pick an interval $q$ as a first guess for an interval which contains $\bar{t}$ and such that $i(t)$ is continuously differentiable on $q$. Let $s_{0}$ be the left end-point of $q$.

Step 2. Calculate $f\left(s_{0}, w\right)$ and $f\left(s_{0}, T\right)$ where $f(t, x):=\frac{e^{-t}}{e^{x}-1} \int_{t}^{t+x} I(s) e^{s} d s$.

Step 3. $\dot{V}\left(s_{0}^{+}\right)=\frac{1-e^{-w}}{w}\left(f\left(s_{0}, w\right)-f\left(s_{0}, T\right)\right)$.

Step 4. Let $\hat{t}=s_{0}+\ln \left(1-\frac{\dot{V}\left(s_{0}^{+}\right)}{\dot{i}\left(s_{0}^{+}\right)}\right)$. If $\hat{t} \in q$ then $\bar{t}=\hat{t}, p=q$, and $t_{0}=s_{0}$. If $\hat{t} \notin q$, then go to Step 1 .

Step 5 . The no spiking condition is given by: $i\left(t_{0}\right)+\dot{i}\left(t_{0}^{+}\right)\left(\bar{t}-t_{0}\right)<1$. 
In Lemma 4.2 and Theorems 4.3 through 4.5, we provide the proof of the above algorithm. Depending on the input current $i(t)$, the readout neuron can be either in repetitive firing mode or quiescent mode. We wish to find conditions on the input current $i(t)$ or alternatively on the parameters $\alpha, \beta, c, h, T, w$ which determine the mode of firing or quiescence.

Lemma 4.2. Let $t_{\text {sim }}$ denote a (sufficiently long) time period of simulation. The readout neuron fires no spikes after some initial time period $t_{\text {init }}<t_{\text {sim }}$ if and only if $V_{\text {max }}<V_{\text {thresh }}$ where $V_{\text {max }}$ is the maximum value of the voltage over $\left(t_{\text {init }}, t_{\text {sim }}\right)$.

Proof. Suppose that the neuron is in quiescence mode. In this case the voltage satisfies the following ordinary differential equation (8) where the input current is given by (9).

$$
\begin{gathered}
\dot{V}(t)=-V(t)+i(t) \\
i(t)=\frac{1}{w} \int_{t}^{t+w} I(u) d u, \text { where } \\
I(t)= \begin{cases}\alpha, & \text { if } t(\bmod T) \in(0,+c) \\
-\beta, & \text { if } t(\bmod T) \in(c, c+h) \\
0, & \text { if } t(\bmod T) \in(c+h, T)\end{cases}
\end{gathered}
$$

The particular solution for any initial condition converges to the steady state solution which is a unique solution with period $T$, the period of the input current. Because the neuron is quiescent we must have $V_{\max }<V_{\text {thresh }}$.

Conversely if for all $t_{\text {init }}, V_{\text {max }}\left(t \in\left(t_{\text {init }}, t_{\text {sim }}\right)\right) \geq V_{\text {thresh }}$, then the neuron is in repetitive firing mode.

Lemma 4.2 tells us that to identify the parameter regime for quiescence, we only need to solve (8) and (9) and impose the condition that for all time $t, V(t)<V_{\text {thresh }}$.

Theorem 4.3. The general solution to (8) is

$$
V(t)=e^{-t}\left(\int_{x}^{t} e^{u} i(u) d u+V(x) e^{x}\right) \quad \text { for all } x \in \mathbb{R}
$$

and the particular solution with the periodicity condition $V(t+T)=V(t)$ is

$$
V(t)=\frac{e^{-t}}{e^{T}-1} \int_{t}^{t+T} e^{u} i(u) d u
$$

Proof. The expression in (10) is a well-known result. Replace $x$ by $t$ and $t$ by $t+T$ in (10) to get $V(t)=$ $V(t+T)=e^{-(t+T)}\left(\int_{t}^{t+T} e^{u} i(u) d u+V(t) e^{t}\right)$. Solve for $V(t)$ to get (11).

Theorem 4.4. Let $f(t, x):=\frac{e^{-t}}{e^{x}-1} \int_{t}^{t+x} I(s) e^{s} d s$. The particular solution to (8) and (9) with the periodicity condition is given by either one of the following equivalent forms

$$
\text { 1. } V(t)=\frac{e^{-t}}{w\left(e^{T}-1\right)} \int_{t}^{t+T} \int_{u}^{u+w} e^{u} I(s) d s d u \text {. }
$$




$$
\begin{aligned}
& \text { 2. } V(t)=i(t)+\frac{1-e^{-w}}{w}(f(t, T)-f(t, w)) \\
& \text { 3. } \dot{V}(t)=\frac{1-e^{-w}}{w}(f(t, w)-f(t, T))
\end{aligned}
$$

Proof. The first equation is a result of substituting (9) into (11). Let $\sigma_{x}:=e^{x}-1$. In order to derive the second equation, we change the order of integration and split the region of integration into (i) $t \leq u \leq s, t \leq$ $s \leq t+w$, (ii) $s-w \leq u \leq s, t+w \leq s \leq t+T$, and (iii) $s-w \leq u \leq t+T, t+T \leq s \leq t+T+w$. We integrate with respect to $u$ to obtain

$$
\begin{aligned}
& V(t)=\frac{e^{-t}}{w \sigma_{T}}\left[\int_{t}^{t+w} I(s)\left(e^{s}-e^{t}\right) d s+\int_{t+w}^{t+T} I(s) e^{s}\left(1-e^{-w}\right) d s+\int_{t+T}^{t+T+w} I(s)\left(e^{t+T}-e^{s-w}\right) d s\right] \\
& =\frac{e^{-t}}{w \sigma_{T}}\left[-\sigma_{T-w} \int_{t}^{t+w} I(s) e^{s} d s+e^{t} \sigma_{T} \int_{t}^{t+w} I(s) d s+e^{-w} \sigma_{w}\left(\int_{t}^{t+T}-\int_{t}^{t+w}\right) I(s) e^{s} d s\right] \\
& =i(t)+\frac{1-e^{-w}}{w}\left[\frac{e^{-t}}{\sigma_{T}} \int_{t}^{t+T} I(s) e^{s} d s-\frac{e^{-t}}{\sigma_{w}} \int_{t}^{t+w} I(s) e^{s} d s\right]
\end{aligned}
$$

where in the second step we used the periodicity condition $I(t+T)=I(t)$. The final step is the desired equation. Finally, the third equation is simply a consequence of the differential equation $\dot{V}(t)=-V(t)+$ $i(t)$.

Theorem 4.5. Let $\bar{t}$ be such that $V(\bar{t}) \geq V(t)$ for all $t$. Let $p$ be an interval containing $\bar{t}$ where $i(t)$ is continuously differentiable and let $t_{0}$ be the left end-point of $p$. Then

$$
V(\bar{t})=i\left(t_{0}\right)+\dot{i}\left(t_{0}^{+}\right) \ln \left(1-\frac{\dot{V}\left(t_{0}^{+}\right)}{\dot{i}\left(t_{0}^{+}\right)}\right)
$$

Proof. $i(t)$ and $V(t)$ are continuously differentiable on $p$. The maximum of $V(t)$ occurs when $\dot{V}(t)$ crosses 0 from above. Based on the form of $i(t)$, it is not too difficult to see that this occurs exactly once during any one period and for a value of $\bar{t} \in(c-w, c)$. On each interval $q$ on which $i(t)$ is continuously differentiable, $i(t)$ has the form $\lambda+\mu t$. The general solution on $q$ is $V(t)=\lambda+\mu(t-1)+\gamma e^{-t}$, where $\gamma$ is an undetermined constant. So $\dot{V}(\bar{t})=0$ implies that $\bar{t}=\ln \left(\frac{\gamma}{\mu}\right)$ and

$$
V(\bar{t})=i(\bar{t})=\lambda+\mu \ln \left(\frac{\gamma}{\mu}\right)
$$

From the initial condition, $V\left(t_{0}\right)=\lambda+\mu\left(t_{0}-1\right)+\gamma e^{-t_{0}}$, we get $\gamma=\left(V\left(t_{0}\right)-i\left(t_{0}\right)+\mu\right) e^{t_{0}}=\left(-\dot{V}\left(t_{0}^{+}\right)+\right.$ $\dot{i}(t)) e^{t_{0}}$. Plugging this into (7) gives (6).

This completes the proof of the algorithm. We demonstrate the algorithm by carrying out an explicit calculation for a couple of cases. In the following, let $\sigma_{x}:=e^{x}-1$. 
I) $0<w \leq c$.

In the interval $t \in(c-w, c)$,

$$
i(t)=\frac{c}{w}(\alpha+\beta)-\beta-\frac{1}{w}(\alpha+\beta) t
$$

Steps 2 and 3 of the algorithm tell us that

$$
\dot{V}(c-w)=\frac{\sigma(w)}{w \sigma(T)}[\alpha \sigma(T-c)+\beta \sigma(h)], \quad i(c-w)=\alpha
$$

Finally Step 5 of the algorithm gives the explicit no spiking condition

$$
\alpha-\frac{\alpha+\beta}{w} \ln \left[1+\frac{\sigma(w)}{\sigma(T)}\left(\frac{\alpha \sigma(T-c)+\beta \sigma(h)}{\alpha+\beta}\right)\right]<1
$$

II) $c<w \leq h$. In the interval $t \in(c-w, c)$,

$$
i(t)=\frac{c}{w}(\alpha+\beta)-\beta-\frac{1}{w}\left(\alpha H_{0}(t)+\beta\right) t
$$

where $H_{0}(t)=1$ if $t \geq 0$ and 0 otherwise.

$$
\dot{V}(c-w)=\frac{1}{w}\left(\alpha e^{-c} \sigma(c)\left(1-\frac{\sigma(w)}{\sigma(T)}\right)+\beta \frac{\sigma(w) \sigma(h)}{\sigma(T)}\right), \quad i(c-w)=\frac{c \alpha}{w}
$$

(a) By way of Step 1 of the algorithm, pick $q=(c-w, 0)$. Steps 4 and 5 of the algorithm gives

$$
\begin{aligned}
& \hat{t}=c-w+\ln \left[1+\frac{\sigma(w)}{\beta \sigma(T)}\left(\alpha e^{-c} \sigma(c)\left(\frac{\sigma(T)}{\sigma(w)}-1\right)+\beta \sigma(h)\right)\right] \\
& \frac{c \alpha}{w}-\frac{\beta}{w} \ln \left[1+\frac{\sigma(w)}{\beta \sigma(T)}\left(\alpha e^{-c} \sigma(c)\left(\frac{\sigma(T)}{\sigma(w)}-1\right)+\beta \sigma(h)\right)\right]<1
\end{aligned}
$$

For sufficiently large $\alpha$, say $\alpha>w / c(\beta+1)-\beta$, combining the two previous equations results in $\hat{t}>0$ and so $p \neq(c-w, 0)$.

(b) We return to Step 1 of the algorithm and pick $q=(0, c)$, Step 5 of the algorithm gives

$$
\alpha-\frac{\alpha+\beta}{w} \ln \left[1+\frac{\sigma(w)}{\sigma(T)}\left(\frac{\alpha \sigma(T-c)+\beta \sigma(h)}{\alpha+\beta}\right)\right]<1
$$

which is the same condition as that found for $0<w \leq c$.

We are now ready to prove the main theorem.

Theorem 4.1 (No Spiking condition). We recast one of the expressions (14) or (17) into the original dimensional form and define the synchrony parameter $s:=1-w / T$. 